\title{
Hepatitis A: who in NSW is most at risk of infection?
}

\author{
Kate Ward ${ }^{\mathrm{A}, \mathrm{C}}$ and Jeremy McAnulty ${ }^{\mathrm{B}}$ \\ ${ }^{\mathrm{A}} N S W$ Public Health Officer Training Program, \\ NSW Department of Health \\ ${ }^{\mathrm{B}}$ Communicable Diseases Branch, NSW Department of Health \\ ${ }^{\mathrm{C} C o r r e s p o n d i n g ~ a u t h o r . E m a i l: ~ k w a r d @ d o h . h e a l t h . n s w . g o v . a u ~}$
}

\begin{abstract}
The incidence of hepatitis A in NSW has declined in recent years, but the relative importance of risk factors remains unclear. Methods: We analysed case data from the NSW Notifiable Diseases Database from 1991 to 2006 . Results: Hepatitis A notification rates fell from 18.9 to 1.4 cases per 100000 between 1991 and 2006 . International travel to endemic areas was the likely exposure for 50 per cent of cases between 2002 and 2006. Rates were five times higher in travellers born in countries where hepatitis A is endemic compared with those born in Australia. Conclusion: Travellers born in endemic countries should be carefully assessed for vaccination before departure.
\end{abstract}

Hepatitis A causes considerable morbidity worldwide with an estimated annual total of 1.5 million clinical cases. ${ }^{1}$ Hepatitis A infection is primarily spread by the faecal oral route. In children, infection is usually asymptomatic but in adults it can cause fever, malaise, anorexia, nausea and/or abdominal discomfort followed by jaundice. Lifelong immunity develops following infection. ${ }^{2}$ Recent seroprevalance studies report declining rates of naturally acquired immunity in some countries where the disease is endemic, presumably due to significant improvements in sanitation. ${ }^{3-5}$

In NSW in the 1990s, several outbreaks of hepatitis A were reportedly associated with male-to-male sexual contact and illicit drug use, and in 1997 a large outbreak was associated with the consumption of raw oysters. ${ }^{6,7}$

A highly effective vaccine was introduced in the 1990s and is recommended for: all people travelling to moderate to high endemic areas; those with intellectual disabilities or chronic liver disease; and those whose occupation or lifestyle may increase their risk of acquiring the infection. ${ }^{8}$ It is likely that the introduction of a commercially avail- able vaccine has contributed to the overall reduction of hepatitis A in NSW; however, other factors contributing to the decline remain unclear.

Uptake of immunisation among Australian travellers to areas where hepatitis A is endemic is reportedly low. ${ }^{9}$ Other research has reported travel to endemic areas from low risk countries as an important risk factor for acquiring hepatitis A infection. ${ }^{10-13}$ Recent data from England and Wales indicates that a higher rate of hepatitis A is observed in South-Asian-born residents, with the majority of these cases acquiring their infection while travelling to areas where hepatitis $\mathrm{A}$ is endemic. ${ }^{14}$

The aim of the present study was to investigate the changing incidence of hepatitis A in NSW from 1991 to 2006, and the relative importance of several risk factors from 2002 to 2006.

\section{Methods}

Under the NSW Public Health Act 1991, doctors, hospitals and laboratories must notify NSW Health of cases of viral hepatitis. Detection of anti-hepatitis A IgM, in the absence of recent vaccination, or the detection of hepatitis A virus by nucleic acid testing is required to confirm a diagnosis of hepatitis A. Since 1991, public health unit staff have investigated notified cases and recorded basic demographic and disease details onto the NSW Notifiable Diseases Database (NDD). In 2002, routine surveillance data collection was enhanced to include information on exposure to risk factors for acquiring the disease between two and seven weeks before the onset of symptoms (incubation period). These risk factors included: recreational drug use; male-to-male sexual contact; travel to endemic areas (by the case or a household member); contact with another possible or notified case; attendance at child care; contact with raw sewerage; and consumption of shellfish. The country where most time was spent was identified for those cases that reported a travel history during their incubation period. It was assumed for cases reporting travel to an endemic country for any length of time during their exposure period that hepatitis A was most likely acquired overseas. Countries were considered endemic on the basis of the World Health Organization classification as shown in Figure $1 .{ }^{15}$ Estimates for the NSW population born in Australia and in endemic countries were obtained using 2006 census data.

\section{Results}

In NSW, hepatitis A notification rates fell from 18.9 cases per 100000 in 1991 to 1.4 cases per 100000 in 2006 


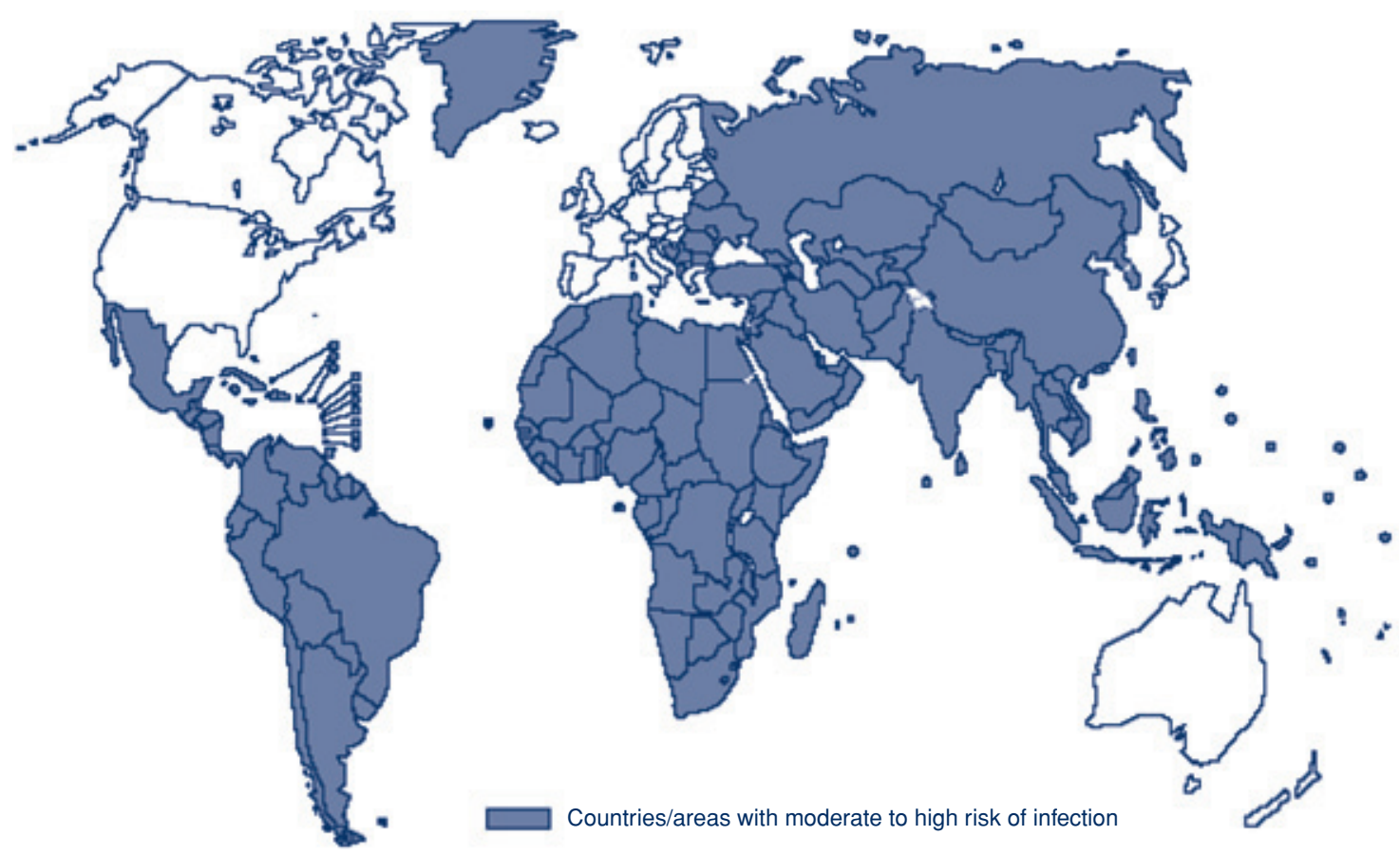

Figure 1. Worldwide distribution of hepatitis A endemicity, 2003

Source:World Health Organization. Hepatitis A vaccine. http://www.who.int/vaccines/en/hepatitisa.shtml

(Figure 2). The age of cases was consistent over time with a mean of 29.9 years in 1991 and 30.9 years in 2006. The proportion of cases that were male decreased from 81 per cent in 1991 to 48 per cent in 2006 .

From 2002 to 2006, 586 cases were notified to NSW Health. For only 8 per cent of these cases was the field identifying whether the person was an Aboriginal or Torres Strait Islander person completed; of these 2 per cent were Aboriginal or Torres Strait Islander people. Information on vaccination status was complete for 49 per cent of cases. Of the cases where vaccination status was documented, 94 per cent were unvaccinated.

Information on risk factors was available for 78 per cent $(n=458)$ of cases. Of these, the most commonly reported risk factor was international travel to endemic areas (Table 1) and all other risk factors were less commonly reported.

Travel to endemic areas was associated with the highest number of notifications each year from 2002 to 2006 with

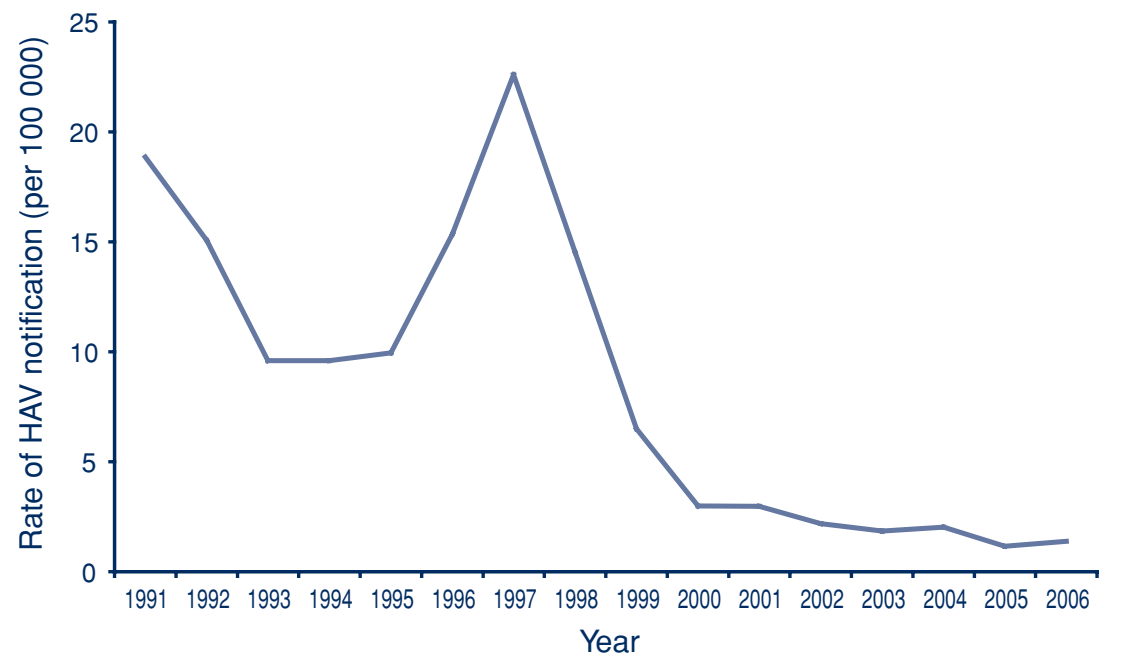

Figure 2. Hepatitis A notification rates from 1991 to 2006 in NSW. Source: NSW Notifiable Diseases Database, NSW Health. 
Table 1. Frequency of risk factors reported by hepatitis A cases in NSW, 2002 to 2006

\begin{tabular}{lrr}
\hline Risk factor* & $\boldsymbol{n}$ & $\%$ \\
\hline Travel to endemic areas & 228 & 50 \\
Household member travel to endemic area & 121 & 26 \\
Contact with another notified case & 44 & 10 \\
Contact with another possible case & 42 & 9 \\
Male-to-male sexual contact & 31 & 7 \\
Ate raw shellfish & 30 & 7 \\
Contact with raw sewerage & 17 & 4 \\
Recreational drug use & 16 & 3 \\
Attends child care centre & 5 & 1 \\
\hline *Categories are not mutually exclusive and in many instances data \\
was incomplete. \\
Source: NSW Notifiable Diseases Database.
\end{tabular}

an average of 46 notifications per year (range: 37-56). This increased as a proportion of all reported cases with risk factor information from 35 per cent (37/107) in 2002 to 65 per cent (53/81) in 2006. For the 96 per cent of cases for whom the country of travel was documented, South and South-East Asia were the most commonly reported travel destinations (Figure 3).

Information on place of birth was available for 89 per cent of cases reporting travel to endemic areas in their exposure period. By place of birth, the rate of hepatitis A in travellers born in endemic countries was significantly greater $(p<0.0001)$ than the rate in Australian-born travellers (12.5 cases per 100000 and 2.3 cases per 100000 , respectively) (Figure 4). Cases born where hepatitis A is endemic primarily originated from South and South-East Asian countries (Table 2) and 90 per cent of cases acquired the infection while returning to their country of birth.

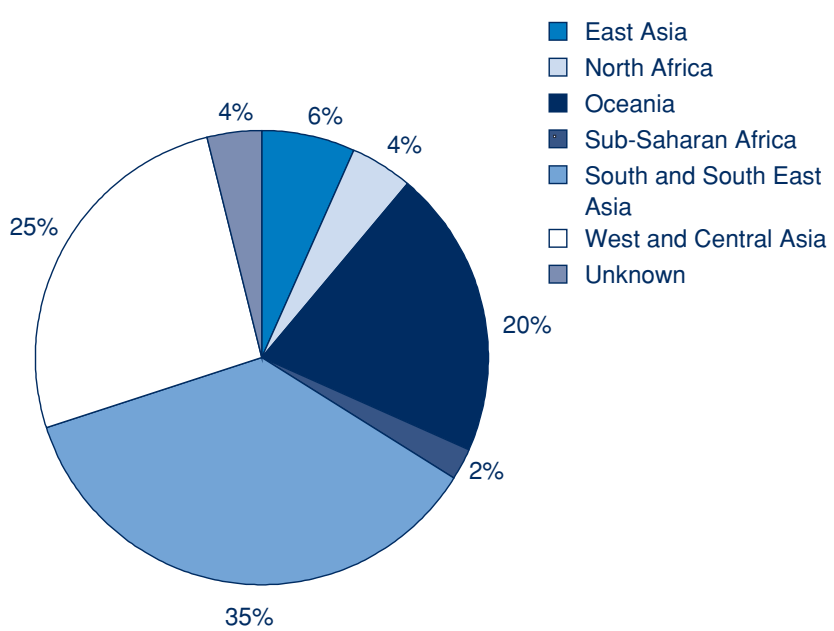

Figure 3. Likely geographical region where hepatitis A was acquired as reported by cases in NSW, 2002 to 2006. Source: NSW Notifiable Diseases Database.

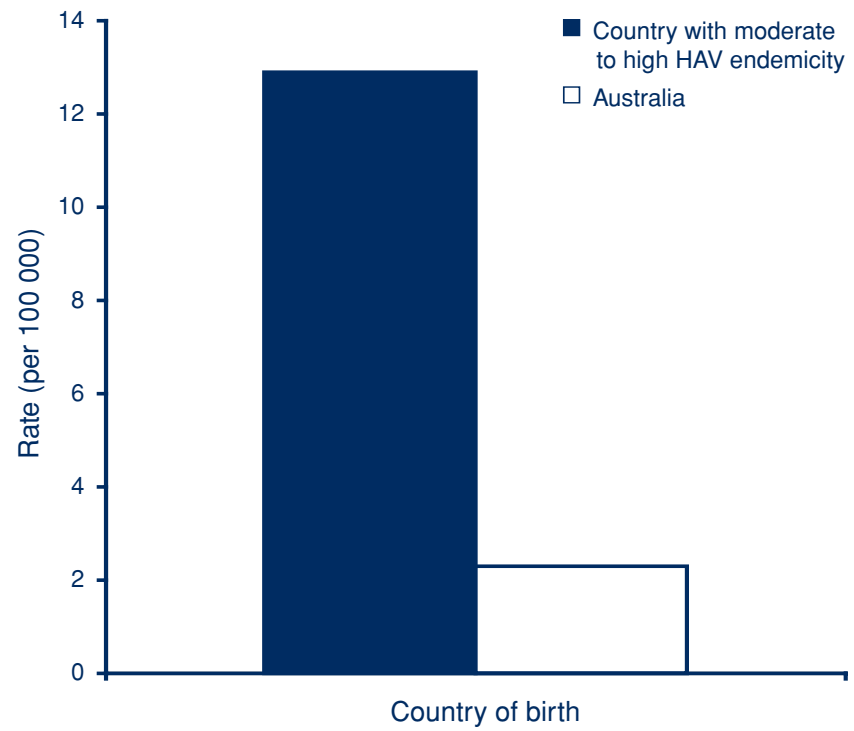

Figure 4. Rate of hepatitis A infection associated with international travel to endemic areas for persons born in Australia compared with those born in countries with moderate to high endemicity.

Source: NSW Notifiable Diseases Database.

\section{Discussion}

In recent years, the rate of hepatitis A has declined dramatically in NSW. While analysis of outbreak data indicates that in the 1990s male-to-male sexual contact and recreational drug use were important risk factors, these are now associated with only a small number of cases. ${ }^{6}$ The dramatic reduction in the proportion of male cases from 81 per cent in 1991 to 48 per cent in 2006 is most likely a result of a decline in transmission between men who have sex with men in this period. Although there is no evidence that the absolute number of cases acquired during travel to endemic areas has increased, it is now the most common risk factor for hepatitis A cases in NSW.

Given the growing proportion of cases with hepatitis A reporting travel to endemic countries and the popularity of South Asian travel destinations for Australian travellers, efforts to promote pre-travel vaccination are increasingly important to reduce the burden of disease in Australia. ${ }^{9}$ In

Table 2. Global region of birth for NSW hepatitis A cases associated with travel, 2002 to $2006(n=203)$

\begin{tabular}{lc}
\hline Region of birth & Proportion (\%) \\
\hline Australia & 52 \\
South and South East Asia & 21 \\
West and Central Asia & 10 \\
Europe & 5 \\
East Asia & 5 \\
Oceania & 5 \\
Sub-Saharan Africa & 2 \\
\hline Source: NSW Notifiable Diseases Database. &
\end{tabular}


addition, given the infectiousness of hepatitis A before the onset of symptoms and diagnosis, vaccination of travellers is the most efficient means of preventing secondary cases in household members.

The present study is limited by the data available on the vaccination status of reported cases being incomplete. However, given the high vaccine efficacy reported in other studies, and low vaccine uptake reported in Australian travellers, it is reasonable to assume that the majority of notified cases were unvaccinated. Information on other risk factors was also incomplete. Self-reporting of behaviours such as male-to-male sexual contact and recreational drug use may underestimate the true prevalence of these risk factors but there is no evidence to suggest that underreporting varied over the period. More complete case information, including risk factors and vaccination status, would enhance understanding of the epidemiology of hepatitis A.

Compared with Australian-born travellers, travellers born in endemic countries returning to their country of origin are at increased risk of acquiring hepatitis A infection. ${ }^{14}$ Factors that may influence the likelihood of overseas-born travellers acquiring natural immunity to hepatitis $\mathrm{A}$ in childhood include the country and region of origin, their socioeconomic status and the age they left the endemic area.

In highly endemic countries, most people experience asymptomatic infection within the first few years of life. ${ }^{2}$ However, the declining risk of hepatitis A transmission in some endemic countries, primarily due to improved sanitation and standards of living, has resulted in a decreased risk of infection in early childhood. ${ }^{1}$ Subsequently, travellers returning to their country of birth to visit family and friends living in areas with poorer sanitation may be at greater risk of hepatitis A than other tourists staying in hotels and dining in restaurants. Furthermore, travellers born in endemic areas who are returning 'home' may be less likely to seek travel advice before departure and more likely to have repeated and/or longer visits, increasing their overall exposure to risk of disease. A recent seroprevalence study involving Indian- and Chinese-born immigrants to the United States recommends assessing immunity before travel for younger immigrants given their greater susceptibility to infection. ${ }^{16}$

The NHMRC immunisation handbook currently recommends screening for pre-existing immunity in those individuals who spent their early childhood in endemic areas. ${ }^{8}$ Economic analyses are required to compare the value of pre-vaccination screening to vaccination alone for endemic born travellers to endemic areas.

Based on NSW data, travellers born in countries where hepatitis A is endemic who are now residing in Australia may not have immunity to hepatitis A infection and should be carefully assessed for vaccination before departure to endemic areas.

\section{References}

1. World Health Organization. Hepatitis A vaccine. Geneva: World Health Organization http://www.who.int/vaccines/ en/hepatitisa.shtml accessed July 2007.

2. Koff RS. Hepatitis A Lancet 1998; 351(9116): 1643-9. doi:10.1016/S0140-6736(98)01304-X

3. Chatproedprai S, Chongsrisawat V, Chatchatee P, Theamboonlers A, Yoocharoen P et al. Declining trend in the seroprevalence of infection with hepatitis A virus in Thailand. Ann Trop Med Parasitol 2007; 101(1): 61-8. doi:10.1179/136485907X157040

4. Almuneef MA, Memish ZA, Balkhy HH, Qahtani M, Alotaibi B, Hajeer A et al. Epidemiologic shift in the prevalence of Hepatitis A virus in Saudi Arabia: a case for routine Hepatitis A vaccination. Vaccine 2006; 24(27-28): 5599-603. doi:10.1016/j.vaccine.2006.04.038

5. Sacy RG, Haddad M, Baasiri G, Khoriati A, Gerbaka BJ, Abu-Elyazeed R. Hepatitis A in Lebanon: a changing epidemiological pattern. Am J Trop Med Hyg 2005; 73(2): 453-6.

6. Delpech VC, Thackway SV, Young L, Pontivivo G, Smedley E, Morgan K, Ferson MJ. Hepatitis A in south-eastern Sydney 1997-1999: continuing concerns for gay men and an outbreak among illicit drug users. Commun Dis Intell 2000; 24(7): 203-6.

7. Conaty S, Bird P, Bell G, Kraa E, Grohmann G, McAnulty JM. Hepatitis A in New South Wales, Australia from consumption of oysters: the first reported outbreak. Epidemiol Infect 2000; 124(1): 121-30. doi:10.1017/S0950268899003386

8. National Health and Medical Research Council. Australian Immunisation Handbook. 8th edn. Canberra: National Health and Medical Research Council 2003.

9. Zwar N, Streeton CL, Travel Health Advisory Group. Pretravel advice and hepatitis A immunization among Australian travelers. J Travel Med 2007; 14(1): 31-6. doi:10.1111/j.17088305.2006.00088.x

10. Mutsch M, Spicher VM, Gut C, Steffen R. Hepatitis A virus infections in travelers, 1988-2004. Clin Infect Dis 2006; 42(4): 490-7. doi:10.1086/499816

11. Steffen R. Changing travel-related global epidemiology of hepatitis A. Am J Med 2005; 118: 46-9. doi:10.1016/j.amjmed. 2005.07.016Suppl 10A

12. Pollock SL, Sheikholeslami A, Edgar B, David ST, Buxton JA. The changing epidemiology of hepatitis A in British Columbia: using health authority follow-up data to inform policy and practice. Can Commun Dis Rep 2006; 32(20): 239-44.

13. Frank C, Walter J, Muehlen M, Jansen A, van Treeck U, Hauri AM et al. Major outbreak of hepatitis A associated with orange juice among tourists, Egypt, 2004. Emerg Infect Dis 2007; 13(1): 156-8.

14. Gungabissoon U, Andrews N, Crowcroft NS. Hepatitis A virus infection in people of South Asian origin in England and Wales: analysis of laboratory reports between 1992 and 2004. Epidemiol Infect 2007; 135(4): 549-54. doi: $10.1017 /$ S0950268806007242

15. World Health Organization. International Travel and Health 2007. Geneva: World Health Organization, 2007. Available from http://www.who.int/ith/en/ accessed July 2007.

16. Ooi WW, Gallagher A, Chen LH. Immunity to hepatitis A and hepatitis B in Indian and Chinese immigrants seen in a travel clinic in Massachusetts, United States. J Travel Med 2006; 13(4): 212-8. doi:10.1017/S0950268806007242 